\section{Percepção, conhecimento e uso de medicamentos genéricos no Sul do Brasil: o que mudou entre 2002 e $2012 ?$}

\author{
Perception, knowledge, and use of generic drugs \\ in southern Brazil: what changed from 2002 \\ to 2012 ?
}

\section{Percepción, conocimiento y uso de medicamentos genéricos en el Sur de Brasil: ¿qué ha cambiado entre 2002 y 2012 ?}

Marília Cruz Guttier 1

Marysabel Pinto Telis Silveira 2

Vera Lucia Luiza 3

Andréa Dâmaso Bertoldi 1

\title{
Resumo
}

Este estudo compara a percepção, conhecimento e uso de medicamentos genéricos em adultos de Pelotas, Rio Grande do Sul, Brasil, por meio de dois estudos transversais de base populacional realizados em 2002 e 2012. Os desfechos estudados foram: (a) prevalência de utilização de medicamentos genéricos; (b) proporção de uso de medicamentos genéricos entre os demais medicamentos; (c) percepção dos usuários sobre preço e qualidade dos medicamentos genéricos; (d) conhecimento dos usuários sobre medicamentos genéricos; $e$ (e) estratégias de aquisição de medicamentos. A prevalência de uso de medicamentos genéricos aumentou de 3,6\% (IC95\%: 3,0-4,3) para 26,1\% (IC95\%: 24,5-27,7) no periodo de dez anos. A percepção sobre preço e qualidade dos medicamentos genéricos se manteve estável, a identificação das características que diferenciam os medicamentos genéricos dos demais medicamentos melhorou $(p<0,001)$ e o erro de classificação de medicamento diminuiu $(p<0,001)$. Houve um aumento significativo na estratégia de aquisição de medicamentos pela substituição do medicamento prescrito pelo medicamento genérico. Entre 2002 e 2012, aumentou o conhecimento e uso de medicamentos genéricos, enquanto a percepção quanto ao menor preço e qualidade equivalente mantiveram-se elevadas.

Conhecimento do Paciente sobre a Medicação; Substituição de Medicamentos; Medicamentos Genéricos

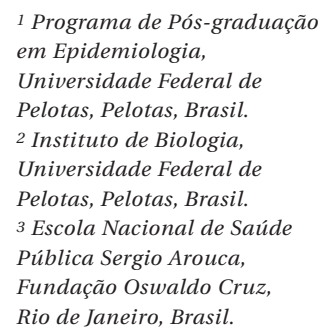

1 Programa de Pós-graduação em Epidemiologia, Universidade Federal de Pelotas, Pelotas, Brasil.

2 Instituto de Biologia Universidade Federal de Pelotas, Pelotas, Brasil. 3 Escola Nacional de Saúde Pública Sergio Arouca, Fundação Oswaldo Cruz Rio de Janeiro, Brasil.

\author{
Correspondência \\ M. C. Guttier \\ Rua Senador Alberto \\ Pasqualini 1061, Pelotas, RS \\ 96080-230, Brasil. \\ maguttier@gmail.com
}




\section{Introdução}

Os medicamentos genéricos foram adotados por vários países como estratégia de ampliação do acesso ao tratamento medicamentoso e de redução dos gastos com medicamentos 1,2. Dados do IMS Health de 2009 sobre o mercado de medicamentos de países de renda alta como Espanha, Alemanha, Reino Unido, Estados Unidos, entre outros, apontavam que os medicamentos genéricos ocupavam entre 30 e $80 \%$ do mercado ${ }^{3}$.

No Brasil, a política dos medicamentos genéricos foi implementada em 1999, pela Lei no 9.7874, com o objetivo de ampliar o acesso a medicamentos com qualidade garantida e preço mais acessível. Desde então, três tipos de medicamentos são comercializados no país: medicamentos de referência (produtos inovadores registrados no órgão federal responsável pela vigilância sanitária e comercializados no país, cuja eficácia, segurança e qualidade foram comprovadas cientificamente junto ao órgão federal competente, por ocasião do registro), medicamentos genéricos (medicamentos designados pela denominação comum brasileira, geralmente produzidos após expiração da proteção patenteária, podendo ser intercambiáveis com os medicamentos de referência) e medicamentos similares (demais medicamentos comercializados com nome comercial que em 2002 e 2012, não considerados intercambiáveis com os medicamentos de referência).

Estudos sobre utilização de medicamentos genéricos realizados na região Sul do Brasil encontraram prevalências de 3,6\% na cidade de Pelotas, Rio Grande do Sul (2002) 5, 9,9\% em Ponta Grossa, Paraná (2007) 6 e 14\% em Tubarão, Santa Catarina (2007) 7.

A confiança nos medicamentos genéricos por parte dos prescritores e usuários é essencial para que aceitem a substituição da prescrição de um medicamento referência por um genérico, e assim, para a ampliação da utilização desses medicamentos ${ }^{8}$. A percepção da população sobre os genéricos tem sido estudada em diferentes países com uso de abordagens tanto quantitativas quanto qualitativas, como mostra a revisão de Hassali et al. 8 .

O conhecimento sobre os genéricos é um aspecto importante que pode influenciar na escolha consciente do produto consumido. Em estudos realizados no Brasil, a proporção daqueles que demonstraram ter conhecimento sobre medicamentos genéricos variou de 42 a $96 \%$ da população. Na maioria desses estudos, o conhecimento foi avaliado pela identificação de características das embalagens ou informações relacionadas ao preço e à qualidade desses medicamentos 5,6,7,9.
O presente estudo tem como objetivo descrever as mudanças na utilização de medicamentos genéricos bem como na percepção e conhecimento da população sobre os mesmos, após 12 anos da entrada desses medicamentos no mercado farmacêutico brasileiro.

\section{Métodos}

Os dados analisados são provenientes de dois estudos transversais de base populacional conduzidos no primeiro semestre dos anos de $2002 \mathrm{e}$ 2012 na cidade de Pelotas, utilizando os mesmos métodos para coleta de dados.

Detalhes sobre o método usado em 2002 estão disponíveis em outro artigo 5 e apenas aspectos relevantes para a comparação com a pesquisa de 2012 serão apresentados aqui. O processo amostral foi idêntico em ambos os estudos, utilizando protocolo de seleção da amostra em múltiplos estágios. A primeira unidade amostral selecionada foi setor censitário e, como unidade amostral secundária, o domicílio. Foram incluídos nos estudos residentes desses domicílios com 20 anos ou mais, exceto aqueles com alguma incapacidade para responder ao questionário.

Realizaram-se cálculos de tamanho de amostra para avaliar as diferenças de proporções dos desfechos em estudo, utilizando poder de $80 \%$, nível de $95 \%$ de confiança e considerando diferenças de 6 a 10 pontos percentuais (p.p.) entre 2002 e 2012 para todos os desfechos. Após acréscimo de $10 \%$ para perdas e recusas e um efeito de delineamento de 2.0, o maior tamanho amostral necessário foi de 2.840 indivíduos.

As medidas foram realizadas nas residências dos indivíduos por entrevistadoras previamente treinadas. Os instrumentos utilizados nos dois estudos foram idênticos e continham questões sobre a utilização de medicamentos nos 15 dias anteriores à entrevista. Para todos os medicamentos utilizados, foi solicitada a apresentação das embalagens e prescrições.

Os medicamentos que tiveram suas embalagens apresentadas foram classificados como genéricos ou não de acordo com suas características oficiais (logomarca e/ou legislação dos genéricos impressos nas embalagens, blisters e bulas). Utilizou-se a classificação internacional Anatomical Therapeutical Chemical (ATC) da Organização Mundial da Saúde (OMS) 10 para classificá-los por grupos farmacológicos.

Estudaram-se os seguintes desfechos nos dois períodos em relação aos medicamentos genéricos: (a) prevalência de uso de pelo menos um genérico; (b) proporção de utilização de genéricos entre os demais medicamentos; (c) per- 
cepção do usuário sobre preço e qualidade dos genéricos; (d) conhecimento sobre as características dos genéricos; e (e) estratégias de aquisição de medicamentos.

Para avaliar a percepção dos usuários sobre os genéricos, foram aplicadas as seguintes questões: (1) o medicamento genérico tem preço maior, igual ou menor em relação ao de marca mais conhecida? e (2) o medicamento genérico tem qualidade melhor, igual ou pior em relação ao de marca mais conhecida?

A avaliação do conhecimento dos usuários sobre os medicamentos genéricos foi medida pela habilidade de reconhecer características das embalagens dos genéricos avaliadas por teste visual e questão objetiva.

O teste visual consistiu-se na apresentação de imagens de embalagens de medicamento de referência, similar e genérico. Primeiramente, foi apresentada a imagem do medicamento de referência para o entrevistado. Em seguida, mostrou-se a imagem da embalagem de um similar e foi perguntado ao entrevistado se era um medicamento genérico (resposta correta = não). Após, foi apresentada a imagem da embalagem do medicamento genérico e, novamente, foi perguntado se o medicamento era genérico (resposta correta $=\operatorname{sim}$ ). Considerou-se erro de classificação aquele que classificou o medicamento similar e/ ou genérico de forma incorreta.

A questão objetiva sobre conhecimento foi: "O que os remédios genéricos possuem nas caixas para que as pessoas saibam que é um medicamento genérico?". Esperou-se o relato espontâneo das características pelos entrevistados. As características diferenciais da embalagem selecionadas foram: lei dos genéricos, letra " $G$ " e a palavra "Genérico".

Adicionalmente, foi criado um escore para análise global dos indivíduos que indicaram conhecimento compatível com o que a legislação preconiza em relação às questões sobre preço menor e qualidade equivalente dos genéricos em relação aos medicamentos de referência e identificação de pelo menos uma das características da embalagem dos medicamentos genéricos. Foram somadas as respostas para as três perguntas e, posteriormente, a variável foi utilizada de forma dicotômica. Atribuiu-se valor um para aqueles que responderam preço menor, qualidade equivalente e identificaram pelo menos uma das características da embalagem e zero para as demais combinações de respostas.

A pergunta sobre a estratégia usual de compra de medicamentos com prescrição médica, presente em ambos os estudos, foi utilizada para identificar as diferenças nas estratégias adotadas pela população para a aquisição de medicamen- tos no início do lançamento dos genéricos no mercado e dez anos após. As possibilidades de respostas foram: (1) compra exatamente o que está na prescrição; (2) substitui o medicamento prescrito apenas se for por um genérico; (3) substitui o medicamento prescrito por medicamento manipulado; (4) substitui o medicamento prescrito pelo remédio que for mais barato, podendo ser genérico, manipulado ou de outra marca.

As variáveis independentes estudadas foram sexo, idade (20-39, 40-59, 60 anos ou mais), escolaridade (0-4, 5-8 e 9 ou mais anos completos de estudo) e classe econômica baseada no Critério de Classificação Econômica Brasileira vigente em cada ano (A são os mais ricos e E os mais pobres) 11,12 .

Para as análises, foram utilizados como denominadores o número total de indivíduos da amostra (cálculo da prevalência de uso de genéricos, percepção sobre as qualificações dos genéricos e estratégias de aquisição de medicamentos) e o número de medicamentos que tiveram suas embalagens apresentadas (proporção de genéricos entre o total de medicamentos utilizados).

Foram realizadas análises descritivas dos desfechos e análises bivariadas utilizando teste de qui-quadrado de heterogeneidade e de tendência linear para comparação de proporções de acordo com as variáveis independentes em cada ano de estudo. Para calcular as razões de prevalências dos desfechos ajustadas para as variáveis em estudo, foi utilizado regressão de Poisson com variância robusta, sendo o valor de p obtido pelo teste de Wald.

Posteriormente, foram analisadas as mudanças nas proporções dos desfechos entre os anos estudados para cada estrato das variáveis independentes pelo teste qui-quadrado de Pearson. Todas as análises levaram em conta o efeito de delineamento amostral pelo uso dos comandos svy do pacote estatístico Stata 12.1 (StataCorp LP, College Station, Estados Unidos).

Este trabalho foi submetido e aprovado pelo Comitê de Ética em Pesquisa da Faculdade de Medicina da Universidade Federal de Pelotas; os indivíduos assinaram termo de consentimento livre esclarecido para participar do estudo.

\section{Resultados}

Em 2002, foram encontradas 3.372 pessoas elegíveis para o estudo, das quais $3.182(94,4 \%)$ responderam à entrevista. Em 2012, a amostra incluiu 3.379 pessoas elegíveis, das quais 2.925 (86,6\%) responderam à entrevista. As perdas e recusas no ano de 2012 foram em maior proporção no sexo masculino $(56,6 \%)$, porém fo- 
ram semelhantes à amostra em relação à média de idade.

A utilização de medicamentos aumentou significativamente entre 2002 e 2012 ( $p<0,001)$. Em 2002, 65,9\% (IC95\%: 64,2-67,5) dos entrevistados utilizavam medicamentos enquanto em 2012, 76,6\% (IC95\%: 75,0-78,1).

A Tabela 1 descreve a amostra, as prevalências e as razões de prevalências ajustadas de utilização de medicamentos genéricos de acordo com as variáveis independentes estudadas. A média de idade foi de 44 anos em 2002 e 45,7 em 2012, sendo observado aumento significativo de idosos na população de Pelotas de 18,3\% em 2002 para 22\% em 2012. Observou-se melhora da situação econômica pelo aumento na frequência de indivíduos classificados na classe A-B de 23,6\% em 2002 para 46,4\% em 2012, e da redução na classe D-E de $36,4 \%$ para $10,1 \%$, respectivamente. Da mesma forma, ocorreu um avanço significativo na escolaridade entre os dois períodos.

Em 2002, a prevalência de utilização de genéricos era de 3,6\% (IC95\%: 3,0-4,3) enquanto em 2012 foi de 26,1\% (IC95\%: 24,5-27,7). Após análise multivariável, constatou-se que em ambos os estudos a utilização de genéricos foi maior entre mulheres e nos grupos de maior idade. Em 2002, a utilização de genéricos aumentou significativamente com a classe econômica $(p=0,021)$ e não houve diferença significativa para escolaridade ( $p=0,118)$. Em 2012, não houve diferença significativa entres as classes econômicas ( $p=0,776)$, mas a prevalência de utilização de genéricos aumentou significativamente com a diminuição da escolaridade ( $\mathrm{p}=0,033)$ (Tabela 1$)$.

A Tabela 2 apresenta a proporção de medicamentos genéricos nos grupos farmacológicos mais utilizados. Em 2002, foram apresentadas as embalagens de 3.352 medicamentos dos 4.609 que foram utilizados (72,7\%). Desses, 3.305 tinham informações sobre ser genérico ou não. Em 2012, foram apresentadas as embalagens de 3.727 medicamentos dos 6.129 que foram utilizados (61\%). Desses, todos tinham informação sobre ser genérico ou não. Os 3.305 e 3.727 medicamentos de 2002 e 2012, respectivamente, foram classificados de acordo com a classificação internacional ATC. As classes terapêuticas que apresentaram maior crescimento relativo de uso de medicamentos genéricos foram: sistema cardiovascular ( $+44,9$ p.p. em 2012), sistema nervoso (+37,8 p.p. em 2012) e antibiótico de uso sistêmico (+33,9 p.p. em 2012).

Ao avaliar a habilidade de reconhecer um medicamento genérico, em 2002, 50,4\% (IC95\%: 48,7-52,2) dos entrevistados erraram a identificação da imagem de um medicamento similar como genérico, enquanto, em 2012, o percentual de erro foi reduzido a 33\% (IC95\%: 31,3-34,7). Em ambos os estudos, a identificação incorreta do medicamento similar como genérico aumentou significativamente conforme a idade $(\mathrm{p}<0,001) \mathrm{e}$ diminuiu com o aumento da escolaridade (2002: $\mathrm{p}<0,001 ; 2012: \mathrm{p}=0,02)$. Em 2002, encontrou-se aumento do percentual de erro de classificação com a diminuição da classe econômica (2002: $p<0,001 ; 2012: p=0,006)$. Em 2012, as mulheres erraram mais a classificação $(p=0,011)$ do que os homens, o que não aconteceu em 2002 ( $\mathrm{p}=0,079)$ (Tabela 3).

Sobre a identificação correta das características da embalagem (lei dos genéricos, letra " $G$ " e a palavra "Genérico"), houve aumento significativo na prevalência de indivíduos capazes de identificar corretamente a embalagem dos medicamentos genéricos. Enquanto em 2002, 56,6\% (IC95\%: 54,9-58,3) identificaram alguma das características da embalagem dos genéricos, em 2012 tal proporção foi de 75,6\% (IC95\%: 74,077,2) (Tabela 3).

Ao avaliar a percepção sobre os medicamentos genéricos por intermédio de questões relativas ao preço e à qualidade em comparação aos de referência, ela se manteve estável durante o período de estudo. Enquanto em 2002, 86\% (IC95\%: 84,7-87,2) dos entrevistados disseram que os medicamentos genéricos custam menos que o medicamento de referência, em 2012 esse percentual foi 87\% (IC95\%: 85,7-88,2). A proporção de entrevistados que acreditavam que os medicamentos genéricos têm qualidade equivalente aos medicamentos de referência foi semelhante em ambos os estudos (em 2002: 70\%, IC95\%: 68,4-71,6; em 2012: 69,1\%, IC95\%: 67,4-70,8) (Tabela 4).

Por outro lado, quando realizadas as comparações por estratos, foi possível identificar diferença significativa na percepção quanto ao preço dos genéricos entre indivíduos de 40 a 59 anos de idade (maior frequência de respostas de preço menor em 2012) ( $p=0,005)$ e entre aqueles das classes D-E (menor frequência de respostas de preço menor em 2012) ( $p=0,044)$. Apresentaram menor frequência de percepção de qualidade equivalente em 2012, os indivíduos de sexo masculino ( $p=0,025$ ), com maior classe econômica $(A-B)(p=0,011)$ e com escolaridade superior a quatro anos de estudo, quando comparados ao período anterior (Tabela 4).

Em 2002, 10,9\% dos entrevistados consideraram a qualidade dos genéricos pior do que os de referência, enquanto em 2012 essa proporção subiu para $16,3 \%$. Verificou-se ainda que, em 2002, não ocorreu diferença na percepção de qualidade dos genéricos entre aqueles que utilizaram e aqueles que não o utilizaram ( $\mathrm{p}=0,447)$. Já em 2012, houve diferença estatisticamen- 
Descrição da amostra, prevalências e razões de prevalências ajustadas de utilização de medicamentos genéricos de acordo com variáveis demográficas e socioeconômicas. Pelotas, Rio Grande do Sul, Brasil, 2002 e 2012.

\begin{tabular}{|c|c|c|c|c|c|c|c|c|c|c|}
\hline \multirow[t]{2}{*}{ Variáveis } & \multicolumn{4}{|c|}{2002} & \multicolumn{4}{|c|}{2012} & \multirow{2}{*}{$\begin{array}{c}\text { Razão } \\
\text { (2012/2002) }\end{array}$} & \multirow{2}{*}{$\begin{array}{c}\text { Valor de } \\
\text { p para } \\
\text { mudança } \\
(2012 / 2002\end{array}$} \\
\hline & n (\%) & $\begin{array}{c}\text { Utilização de } \\
\text { genéricos } \\
\text { (\%) }\end{array}$ & $\begin{array}{l}\text { RP ajustada } \\
\text { (IC95\%) * }\end{array}$ & $\begin{array}{c}\text { Valor } \\
\text { de } p * \star\end{array}$ & n (\%) & $\begin{array}{c}\text { Utilização de } \\
\text { genéricos } \\
\text { (\%) }\end{array}$ & $\begin{array}{l}\text { RP ajustada } \\
\text { (IC95\%) * }\end{array}$ & $\begin{array}{c}\text { Valor } \\
\text { de } p \text { ** }\end{array}$ & & \\
\hline Sexo & & & & 0,006 & & & & $<0,001$ & & \\
\hline Masculino & $1.374(43,2)$ & 2,3 & 1,00 & & $1.202(41,1)$ & 19,1 & 1,00 & & 8,3 & $<0,001$ \\
\hline Feminino & $1.808(56,8)$ & 4,6 & $1,77(1,17-2,68)$ & & $1.723(58,9)$ & 30,9 & $1,53(1,34-1,74)$ & & 6,7 & $<0,001$ \\
\hline Idade (anos) & & & & $<0,001$ & & & & $<0,001$ & & \\
\hline $20-39$ & $1.399(44,0)$ & 1,6 & 1,00 & & $1.152(39,4)$ & 15,6 & 1,00 & & 9,7 & $<0,001$ \\
\hline $40-59$ & $1.200(37,7)$ & 3,8 & $2,34(1,25-4,38)$ & & $1.109(37,9)$ & 25,5 & $1,55(1,29-1,87)$ & & 6,7 & $<0,001$ \\
\hline $\begin{array}{l}60 \text { ou } \\
\text { mais }\end{array}$ & $583(18,3)$ & 7,9 & $4,57(2,37-8,83)$ & & $664(22,7)$ & 45,0 & $2,57(2,16-3,05)$ & & 5,7 & $<0,001$ \\
\hline $\begin{array}{l}\text { Classe } \\
\text { econômica } \\
\star \star \star\end{array}$ & & & & 0,021 & & & & 0,776 & & \\
\hline$A-B$ & $747(23,6)$ & 4,2 & 1,00 & & $1.348(46,4)$ & 24,4 & 1,00 & & 5,8 & $<0,001$ \\
\hline C & $1.270(40,0)$ & 3,5 & $0,75(0,46-1,24)$ & & $1.261(43,5)$ & 26,7 & $1,01(0,87-1,18)$ & & 7,6 & $<0,001$ \\
\hline D-E & $1.153(36,4)$ & 3,2 & $0,55(0,32-0,96)$ & & $294(10,1)$ & 32,3 & $1,03(0,83-1,28)$ & & 10,1 & $<0,001$ \\
\hline $\begin{array}{l}\text { Escolaridade } \\
\text { (anos de } \\
\text { estudo) }\end{array}$ & & & & 0,118 & & & & 0,033 & & \\
\hline $0-4$ & $879(27,7)$ & 5,6 & $1,43(0,89-2,29)$ & & $525(18,0)$ & 39,6 & $1,21(1,02-1,43)$ & & 7,1 & $<0,001$ \\
\hline $5-8$ & $1.067(33,6)$ & 2,8 & $0,99(0,61-1,61)$ & & $817(28,0)$ & 25,7 & $1,04(0,90-1,21)$ & & 9,2 & $<0,001$ \\
\hline 9 ou mais & $1.231(38,7)$ & 2,9 & 1,00 & & $1.580(54,0)$ & 21,6 & 1,00 & & 7,4 & $<0,001$ \\
\hline Total & 3.182 & 3,6 & & & 2.925 & 26,1 & & & 7,3 & $<0,001$ \\
\hline
\end{tabular}

IC95\%: intervalo de 95\% de confiança; RP: razão de prevalência.

* Análise ajustada para sexo, idade, classe econômica e escolaridade;

** Teste de Wald para tendência linear entre as proporções;

*** Indicador construído conforme a Associação Brasileira de Empresas de Pesquisa 11,12.

te significativa na percepção da qualidade dos genéricos entre esses grupos $(p=0,003)$, sendo maior a percepção de que o genérico tem qualidade equivalente entre aqueles que consumiram medicamento genérico do que entre aqueles que não consumiram (dados não apresentados em tabela).

Em uma análise global dos indivíduos que indicaram percepção compatível com o que a legislação preconiza (genéricos têm menor preço e qualidade equivalente aos medicamentos de referência e apresentam características próprias nas embalagens que os diferencia dos demais medicamentos), em 2002, tal proporção foi de 42\% (IC95\%: 40,3-43,7), enquanto em 2012 aumentou para 51,2\% (IC95\%: 49,4-53,1).

A Figura 1 mostra as estratégias que os indivíduos costumam adotar ao comprar medicamentos com prescrição médica evidenciando dife- rença significativa entre os estudos para todas as estratégias estudadas. Existiu diminuição do emprego das estratégias de comprar só o medicamento prescrito, de substituir apenas pelo manipulado ou de substituir pelo mais barato e aconteceu aumento da substituição apenas pelo medicamento genérico.

\section{Discussão}

Este estudo comparou a utilização, a percepção e o conhecimento dos usuários sobre os medicamentos genéricos no início de sua entrada no mercado farmacêutico brasileiro e dez anos após. Pode-se observar um crescimento importante do conhecimento e da utilização de medicamentos genéricos, manutenção de proporções elevadas de percepção sobre preço inferior e qualidade 
Tabela 2

Distribuição dos medicamentos utili zados e proporção de medicamentos genéricos segundo classificação pelo primeiro nível da ATC (Anatomical Therapeutical Chemical Classification 10). Pelotas, Rio Grande do Sul, Brasil, 2002 e 2012.

\begin{tabular}{|c|c|c|c|c|c|c|}
\hline \multirow{2}{*}{$\begin{array}{l}\text { Classes terapêuticas } \\
\text { ATC * }\end{array}$} & \multicolumn{2}{|c|}{$2002 * \star$} & \multicolumn{2}{|c|}{ 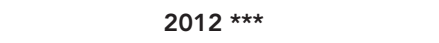 } & \multirow{2}{*}{$\begin{array}{c}\text { Diferença (p.p.) } \\
\text { de utilização de } \\
\text { genéricos (2002-2012) }\end{array}$} & \multirow[t]{2}{*}{ Valor de $p \#$} \\
\hline & $\begin{array}{l}\text { Medicamentos } \\
\text { utilizados ( } n \text { ) }\end{array}$ & $\begin{array}{c}\text { Medicamentos } \\
\text { genéricos [n (\%)] }\end{array}$ & $\begin{array}{l}\text { Medicamentos } \\
\text { utilizados (n) }\end{array}$ & $\begin{array}{c}\text { Medicamentos } \\
\text { genéricos [n (\%)] }\end{array}$ & & \\
\hline $\begin{array}{l}\text { A. Trato alimentar e } \\
\text { metabolismo }\end{array}$ & 463 & $15(3,2)$ & 557 & $169(30,3)$ & $+27,1$ & $<0,001$ \\
\hline $\begin{array}{l}\text { B. Sangue e órgãos } \\
\text { hematopoiéticos }\end{array}$ & 82 & $2(2,4)$ & 131 & $30(22,9)$ & $+20,5$ & $<0,001$ \\
\hline $\begin{array}{l}\text { C. Sistema } \\
\text { cardiovascular }\end{array}$ & 937 & $41(4,4)$ & 1.265 & $623(49,3)$ & $+44,9$ & $<0,001$ \\
\hline $\begin{array}{l}\text { G. Aparelho gênito- } \\
\text { urinário e hormônios } \\
\text { sexuais }\end{array}$ & 288 & $1(0,4)$ & 192 & $7(3,7)$ & $+3,3$ & 0,006 \\
\hline $\begin{array}{l}\text { J. Antibióticos de uso } \\
\text { sistêmico }\end{array}$ & 79 & $15(19,0)$ & 68 & $36(52,9)$ & $+33,9$ & $<0,001$ \\
\hline $\begin{array}{l}\text { M. Sistema } \\
\text { musculoesquelético }\end{array}$ & 357 & $35(9,8)$ & 339 & $82(24,2)$ & $+14,4$ & $<0,001$ \\
\hline N. Sistema nervoso & 703 & $15(2,1)$ & 804 & $321(39,9)$ & $+37,8$ & $<0,001$ \\
\hline R. Sistema respiratório & 134 & $1(0,8)$ & 148 & $29(19,6)$ & $+18,8$ & $<0,001$ \\
\hline $\begin{array}{l}\text { Outros grupos } \\
\text { farmacológicos \#\# }\end{array}$ & 213 & $5(2,3)$ & 205 & $46(22,4)$ & $+20,1$ & $<0,001$ \\
\hline Total \#\#\# & $3.256 \# \# \#$ & $130(3,9)$ & $3.708 \# \# \#$ & $1.343(36,2)$ & $+32,2$ & $<0,001$ \\
\hline
\end{tabular}

* Primeiro nível da classificação internacional das classes terapêuticas baseado na ATC 10;

** Em 2002, dos 4.609 medicamentos utilizados, foram apresentadas as embalagens para 3.352 medicamentos e 3.305 tinham informações sobre ser genérico ou não;

*** Em 2012, dos 6.129 medicamentos utilizados, foram apresentadas as embalagens para 3.727 medicamentos e todos tinham informações sobre ser genérico ou não;

\# Teste qui-quadrado para diferença de proporção de utilização de genéricos dos grupos farmacológicos no período de tempo entre os estudos;

\#\# Outros grupos farmacológicos correspondem a sensorial (S) e dermatológico (D) em 2002 e sensorial (S), dermatológico (D) e hormônio (H) em 2012;

\#\#\# Em 2002, 49 medicamentos que apresentaram a embalagem não foram classificados pela ATC por falta de informação (missing). Em 2012, esse número

foi igual a 19 .

equivalente aos medicamentos de referência, embora alguns grupos analisados tenham indicado pior percepção sobre a qualidade dos genéricos em relação aos medicamentos de referência. Tais achados podem ser indicativos de que a política está evoluindo conforme o planejado. A evolução dessa política amplia o acesso a medicamentos de menor custo, com qualida-de assegurada.

A utilização de medicamentos genéricos em Pelotas foi sete vezes maior em 2012 quando comparada a 2002. Houve importante crescimento na habilidade de diferenciar o medicamento genérico do similar e de reconhecer as características da embalagem. Também foi observado um aumento estatisticamente significativo do número de indivíduos a substituir o medicamento prescrito pelo genérico no momento da compra.

Dados de estudos realizados em 2002 5, 2007 6,7 e 2012 (do presente estudo) indicam que a prevalência de utilização de medicamentos genéricos vem aumentando no Sul do Brasil. Tal crescimento justifica-se em razão da maior disponibilidade e visibilidade desses medicamentos nos estabelecimentos comerciais 13,14 em função do gradual aumento no número de produtos registrados 15,16 e aos preços mais acessíveis 13,17. Estudo realizado no Brasil em 2007, empregando a metodologia de avaliação de disponibilidade e preços proposta pela OMS e Ação Internacional para a Saúde (AIS), apontou que a disponibilidade de genéricos na Região Sul era de $32 \%$ para os medicamentos estudados 14 nas farmácias do setor privado. Já Bertoldi et al. 13 aplicaram a mesma metodologia no Rio Grande Sul em 2008 e encontraram uma disponibilidade de $74 \%$ no setor privado.

Apesar do crescimento da disponibilidade de medicamentos genéricos, não se pode ignorar o 
Conhecimento sobre medicamentos genéricos: erro de classificação de medicamentos similares e/ou genéricos e identificação das características da embalagem de um medicamento genérico estratificado de acordo com variáveis demográficas e socioeconômicas. Pelotas, Rio Grande do Sul, Brasil, 2002 e 2012.

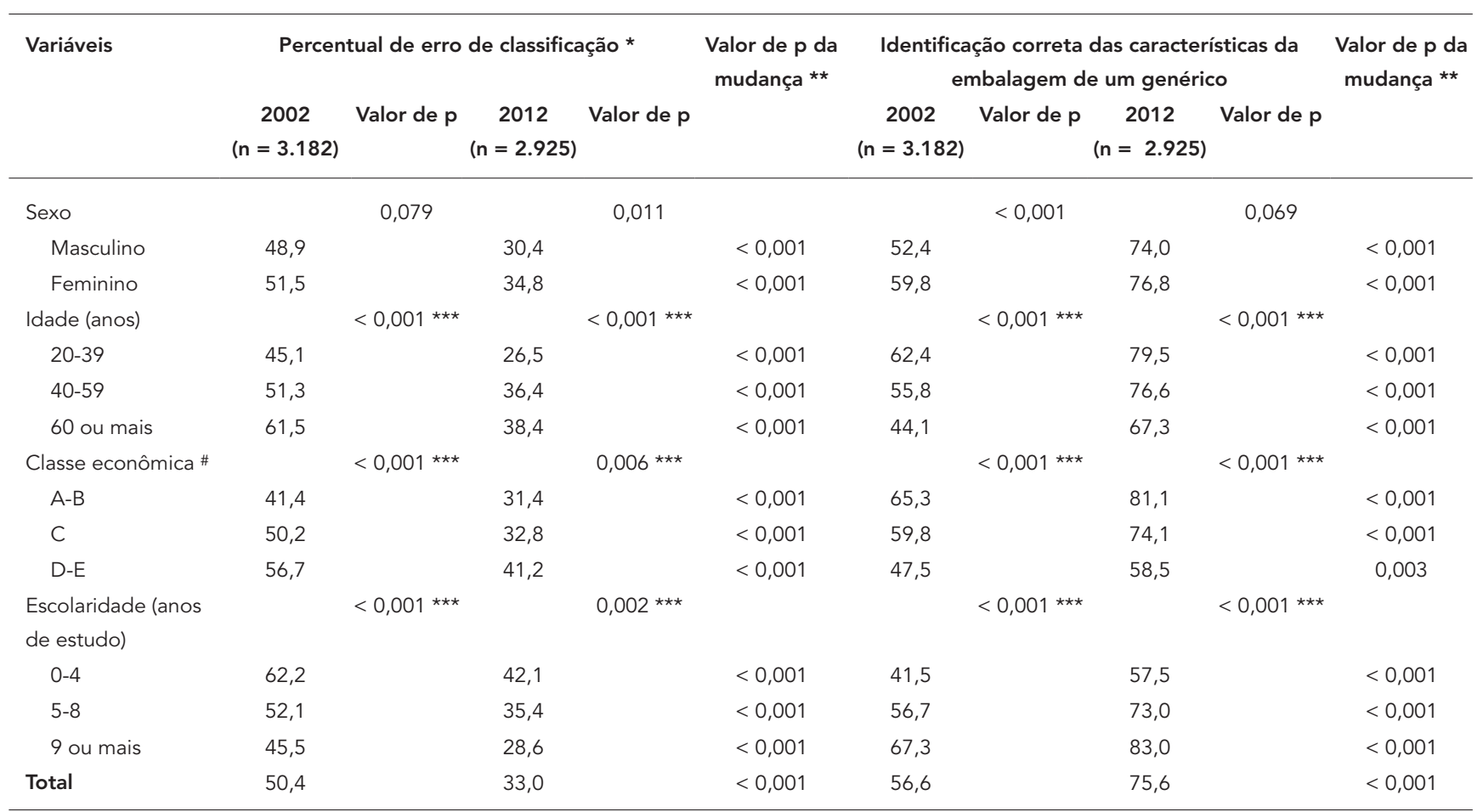

* Foi considerado erro de classificação quando o participante da pesquisa identificou a imagem de um medicamento similar e/ou genérico de forma incorreta;

** Teste do qui-quadrado para diferença de proporções de erro de classificação de medicamentos similares como genéricos no período de tempo entre

os estudos;

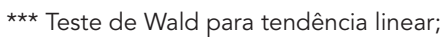

\# Indicador construído conforme a Associação Brasileira de Empresas de Pesquisa 11,12.

fato de que os medicamentos similares, de menor custo e mais disponíveis no serviço público ${ }^{14}$, também são importantes na ampliação do acesso a medicamentos 13. Entretanto, no período de estudo (2002 e 2012) os similares não tinham obrigatoriedade de demonstrar bioequivalência e biodisponibilidade, o que teve início a partir de 2003, com prazo final para adequação de todos os registros em 2014 18,19.

Ao comparar nossos dados de utilização de medicamentos genéricos com o de outros países, percebe-se que o Brasil ainda está aquém dos países que implementaram essa política há mais tempo. Nos Estados Unidos e em alguns países europeus como Alemanha, Espanha e Suécia, onde as seguradoras de saúde subsidiam os gastos com saúde, inclusive com medicamentos, os genéricos ocupam mais de $50 \%$ do volume do mercado farmacêutico ${ }^{3}$.

O Brasil tem grande potencial de exploração do mercado farmacêutico, podendo aumentar a utilização de medicamentos genéricos 20 . Re- latório da IMS Health mostra que os países em desenvolvimento têm apresentado o maior crescimento no mercado farmacêutico por conta das políticas de ampliação de acesso a medicamentos ${ }^{3}$. A demanda por esses produtos, sobretudo pelo governo brasileiro, mediante seus programas e políticas de distribuição de medicamentos à população, estimulou a indústria farmacêutica nacional a focar-se no desenvolvimento, produção e comercialização de medicamentos genéricos e similares 20,21. Estimativas apontam que os medicamentos genéricos serão aqueles que mais crescerão em países de mercado farmacêutico emergente até 2017, como o Brasil, entre outros 22.

Nossos resultados demonstram maior consumo de genéricos entre mulheres, nas maiores faixas etárias e entre pessoas com maior escolaridade, o que também foi constatado no estudo

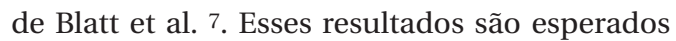
tendo em vista que mulheres procuram mais serviços de saúde, têm mais cuidado com sua saúde e utilizam mais medicamentos que homens de 
Percepção do usuário sobre características de preço e qualidade dos medicamentos genéricos em relação aos medicamentos referência. Pelotas, Rio Grande do Sul, Brasil, 2002 e 2012.

\begin{tabular}{|c|c|c|c|c|c|c|}
\hline \multirow[t]{2}{*}{ Variáveis } & \multicolumn{3}{|c|}{$\begin{array}{c}\text { Considera o preço do genérico menor do que o } \\
\text { medicamento de referência }\end{array}$} & \multicolumn{3}{|c|}{$\begin{array}{c}\text { Considera a qualidade do genérico equivalente a do } \\
\text { medicamento de referência }\end{array}$} \\
\hline & $\begin{array}{c}2002 \\
{[n(\%)]}\end{array}$ & $\begin{array}{c}2012 \\
{[n(\%)]}\end{array}$ & Valor de $p$ * & $\begin{array}{c}2002 \\
\text { [n (\%)] }\end{array}$ & $\begin{array}{c}2012 \\
{[n(\%)]}\end{array}$ & Valor de $p$ * \\
\hline \multicolumn{7}{|l|}{ Sexo } \\
\hline Masculino & $1.162(84,6)$ & $1.038(86,4)$ & 0,265 & $966(70,3)$ & $791(65,8)$ & 0,025 \\
\hline Feminino & $1.574(87,1)$ & $1.507(87,5)$ & 0,752 & $1.262(69,8)$ & $1.231(71,5)$ & 0,328 \\
\hline \multicolumn{7}{|l|}{ Idade (anos) } \\
\hline $20-39$ & $1.264(90,4)$ & $1.017(88,3)$ & 0,142 & $1.060(75,8)$ & $866(75,2)$ & 0,763 \\
\hline $40-59$ & $1.026(85,5)$ & $999(90,1)$ & 0,005 & $829(69,1)$ & $747(67,4)$ & 0,453 \\
\hline 60 ou mais & $446(76,5)$ & $529(79,7)$ & 0,191 & $339(58,2)$ & $409(61,6)$ & 0,258 \\
\hline \multicolumn{7}{|c|}{ Classe econômica ** } \\
\hline$A-B$ & $675(90,4)$ & $1.206(89,5)$ & 0,587 & $569(76,2)$ & $949(70,4)$ & 0,011 \\
\hline C & $1.112(87,6)$ & $1.098(87,1)$ & 0,788 & $897(70,6)$ & $881(69,9)$ & 0,748 \\
\hline$D-E$ & $939(81,4)$ & $222(75,5)$ & 0,044 & $754(65,4)$ & $180(61,2)$ & 0,247 \\
\hline \multicolumn{7}{|c|}{ Escolaridade (anos de estudo) } \\
\hline $0-4$ & $684(77,8)$ & $410(78,1)$ & 0,912 & $492(56,0)$ & $299(57,0)$ & 0,760 \\
\hline $5-8$ & $922(86,4)$ & $706(86,4)$ & 0,999 & $763(71,5)$ & $542(66,3)$ & 0,038 \\
\hline 9 ou mais & $1.128(91,6)$ & $1.426(90,3)$ & 0,260 & $971(78,9)$ & $1.178(74,6)$ & 0,021 \\
\hline Total & $2.736(86,0)$ & $2.545(87,0)$ & 0,381 & $2.228(70,0)$ & $2.022(69,1)$ & 0,566 \\
\hline
\end{tabular}

* Teste qui-quadrado para diferença de proporções entre 2002 e 2012;

** Indicador construído conforme a Associação Brasileira de Empresas de Pesquisa 11,12.

forma geral 23,24. Além disso, o envelhecimento aumenta a prevalência de doenças crônicas não transmissíveis que acarretam maior uso de medicamentos em geral $12 \mathrm{e}$, provavelmente, o aumento do consumo de medicamento genéricos.

Pode-se observar que em 2002 a utilização de medicamentos genéricos aumentava com a melhora da classe econômica e não variava com a escolaridade; já em 2012, a utilização não variou com a classe econômica e diminuiu conforme aumentava a escolaridade. Isso pode ter ocorrido porque em 2002 a utilização dos genéricos ainda estava incipiente; as classes que tinham acesso a informações sobre esses medicamentos eram as A e B, e a educação não fazia diferença na opção pelo uso desses medicamentos. Contudo, dez anos após, percebe-se que a política atinge todas as classes sociais e o foco naqueles mais necessitados (classes D/E e menos escolarizados $0 / 4$ anos de estudo) também está sendo atingido. Esses resultados sugerem que a política dos genéricos está alcançando seus objetivos de ampliação de acesso a medicamentos e promoção da equidade.

A literatura nacional aponta que os medicamentos para doenças crônico-degenerativas são os mais utilizados 25,26 . Verificou-se maior cres- cimento na utilização de medicamentos genéricos entre os grupos farmacológicos que atingem uma parcela ampla da população, como aqueles para o sistema cardiovascular e o sistema nervoso. Entre as doenças agudas, a classe terapêutica que apresentou maior crescimento de utilização de genéricos foi antibióticos de uso sistêmico, sendo os medicamentos mais encontrados nos domicílios ${ }^{7}$. Essas diferenças de utilização de genérico entre as classes terapêuticas pode ser em função da maior diferença de preço entre o de referência e o genérico 27 , maior disponibilidade de medicamentos genéricos no mercado 16 ou por confiança do usuário e do prescritor no genérico dependendo da classe 28 .

Apesar da baixa disponibilidade de medicamentos genéricos no setor público 14,27,29, houve um crescimento importante de programas de assistência farmacêutica no Brasil visando à redução de gastos diretos dos consumidores com medicamentos. Entre eles, está o programa Farmácia Popular do Brasil, iniciado em 200430 e seus planos de expansão lançados posteriormente, como Aqui tem Farmácia Popular e Saúde Não Tem Preço, implantados em $2009{ }^{31}$ e $2011^{32}$, respectivamente. Esses programas podem ter contribuído positivamente para o aumento da 
Diferença a entre as estratégias usuais de compra de medicamentos com prescrição médica. Pelotas, Rio Grande do Sul, Brasil, 2002 e 2012.

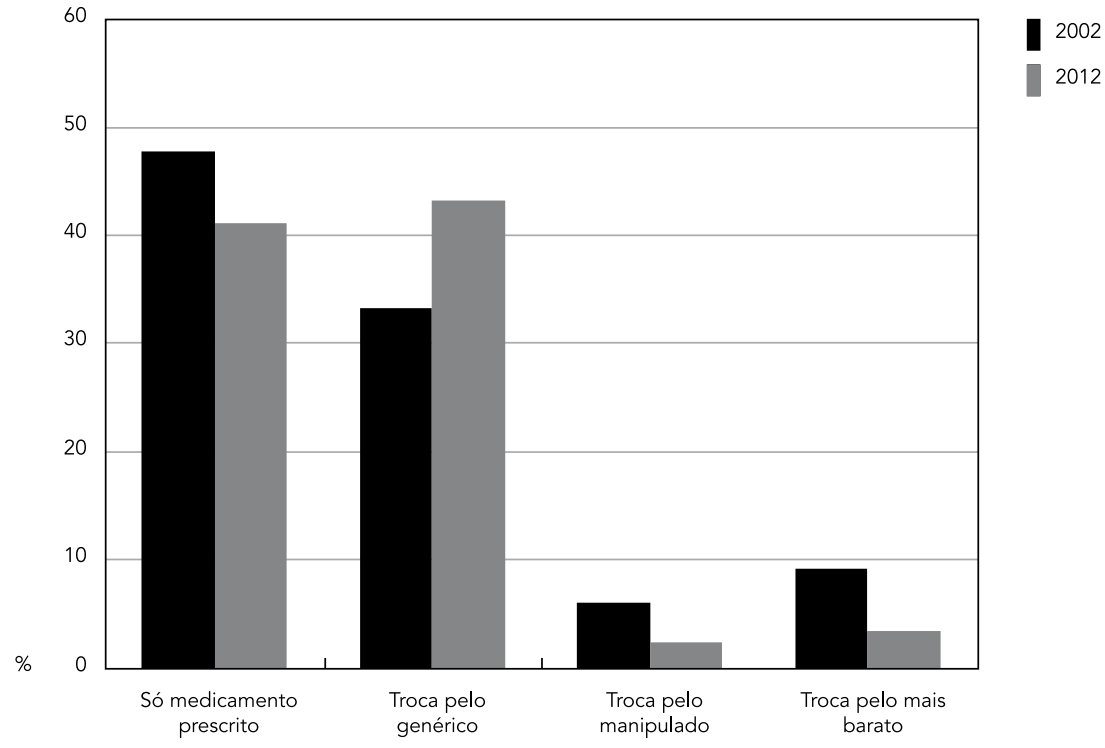

Nota: $p<0,001$ (qui-quadrado entre os dois estudos) para todas estratégias estudadas; a soma das proporções em cada ano não atingem $100 \%$ porque as categorias "nunca compra medicamentos" e "outros" não estão representadas graficamente (correspondendo a 4,4\% em 2002 e 9,7\% em 2012).

utilização de medicamentos genéricos, particularmente os dois últimos, pois estão norteados na redução dos gastos da população com medicamentos e utilizam prioritariamente a distribuição de medicamentos genéricos para ampliar o acesso a medicamentos essenciais na rede privada de farmácias 14,33,34,35,36.

A crença na qualidade dos medicamentos genéricos é um fator que pode influenciar a sua utilização. A diferença de percepção entre usuários e não usuários de medicamentos genéricos com relação à qualidade desses produtos foi significativa apenas em 2012, mostrando que a evolução do conhecimento e a experiência prévia com o genérico pode fazer diferença na percepção de qualidade desse medicamento. Apesar de a grande maioria (70\%) da população acreditar na equivalência entre o medicamento genérico e o de referência, cerca de $30 \%$ da população estudada nos dois períodos dava indícios de não crer na equivalência entre esses medicamentos, o que também foi observado no estudo de Nardi et al. 37 no Brasil e em estudos realizados em outros países, como Jamaica ${ }^{38}$, Malásia 39 e Irlanda 40.

Apesar da percepção sobre as características de preço e qualidade do genérico ter se mantido elevada, encontrou-se um aumento na pro- porção de pessoas que consideram os genéricos de qualidade pior do que os medicamentos de referência. Com o aumento da oferta de medicamentos genéricos, pode ter havido maior variação na qualidade deles por causa da heterogeneidade do processo de produção, uma vez que a legislação permite um desvio da determinação quantitativa do fármaco em matérias-primas ou em formas farmacêuticas de 80 a $120 \%$ nos testes de biodisponibilidade ${ }^{41}$. Por outro lado, estudos têm demonstrado melhor aceitação dos genéricos por aqueles com experiência com esses medicamentos, mas aqueles que experimentaram mostraram boa percepção e aceitação dos medicamentos genéricos 7,42,43.

Houve importante melhora na habilidade de reconhecimento dos medicamentos genéricos, mostrando que os indivíduos estão mais atentos a esses medicamentos e familiarizados com eles, o que também foi constatado em outras pesquisas realizadas no Brasil 6,7,9. Tal melhora na percepção sobre os genéricos pode ter contribuído para mudança nas estratégias usuais de aquisição de medicamentos, aumentando significativamente a substituição de medicamentos prescritos pelos genéricos. 
Atualmente, o crescente número de estudos objetivando estabelecer a relação entre conhecimento sobre os medicamentos genéricos e a sua aceitação tem demonstrado que o médico influencia a aceitação e utilização de medicamentos genéricos e determina, em muitos países, a substituição do tratamento pelo genérico 39,40,44. Estudo de Dunne et al. 40 mostrou que os médicos tendem a manter uma opinião mais negativa dos medicamentos genéricos e transferir essa opinião para seus pacientes, diminuindo a utilização de medicamentos genéricos. Já os farmacêuticos, que também têm papel fundamental na determinação da aceitação dos genéricos, têm boa aceitação e recomendam a intercambialidade sempre que possível 40 .

Pelotas apresentou uma melhora da situação econômica importante no período, sendo uma cidade que apresenta uma distribuição de classes econômicas e escolaridade melhor que a média brasileira 11,12,45,46. Ao serem comparados os dois períodos, a melhora do perfil socioeconômico da população, bem como a ampliação do conhecimento sobre os medicamentos genéricos podem ter contribuído para a busca pela substituição pelo medicamento genérico, reduzindo a busca apenas pelo preço menor. Estudo realizado na Polônia sobre preditores para intercambialidade entre medicamentos genéricos e de referência mostrou que os principais preditores para essa escolha foram a recomendação médica, o conhecimento, a experiência prévia e renda 47 .

Algumas limitações do estudo devem ser apontadas. A proporção maior de perdas no sexo masculino em 2012 pode ter distorcido alguns resultados. Como se teve um percentual mais baixo de uso de genéricos entre os homens, os achados sobre qualidade equivalente dos genéricos e erro de classificação podem estar subestimados. Sobre a identificação correta das características dos genéricos, se fossem incluídos mais homens na amostra poderia ter resultado em uma diferença estatisticamente significativa.

Outra limitação diz respeito à magnitude da diferença da prevalência de utilização entre os dois períodos, pois só foi avaliada a utilização quando a embalagem foi apresentada. Como em 2002 foram apresentadas as embalagens para $72 \%$ dos medicamentos e, em 2012, para $61 \%$, os resultados podem estar subestimando a prevalência de uso. No entanto, a metodologia foi a mesma para ambos os estudos, na intenção de reduzir esse viés.

Em termos de autorrelato sobre a utilização de medicamentos, ele pode levar a erro de recordatório que pode ser minimizado quando se utilizam períodos curtos. No presente estudo comparativo, optou-se por usar um período de recordatório de 15 dias, que é o mais utilizado literatura 48 .

A influência dos programas de assistência farmacêutica como Farmácia Popular, Aqui tem Farmácia Popular e Saúde Não Tem Preço, na ampliação do acesso a medicamentos e da utilização de medicamentos genéricos não foi avaliada por este estudo. Trabalhos futuros poderão esclarecer a magnitude da influência desses programas sobre a ampliação da utilização de medicamentos genéricos.

O aumento significativo na estratégia de aquisição de medicamentos pela substituição do medicamento prescrito pelo genérico entre os dois períodos estudados demonstram que a política de genéricos avançou, ensejando-se maior acesso e maior utilização desses produtos. A discussão de políticas que tornem o medicamento genérico um produto ainda mais competitivo e atraente que os demais medicamentos disponíveis no mercado é necessária e precisa da cooperação das partes interessadas (indústrias, governantes, profissionais de saúde e consumidores). Também uma maior articulação no sentido de estimular a disponibilidade de medicamentos genéricos no serviço público poderia aumentar o sucesso da política dos genéricos. A vigilância sanitária também tem importância fundamental no processo, pois é responsável pela fiscalização para garantia da qualidade, segurança e eficácia desses medicamentos.

Além disso, o uso de estratégia para motivar a prescrição de genéricos pela classe médica, importantes formadores de opinião da população, pode contribuir para aumento da aceitação desses medicamentos, sendo importante para promoção do uso de genéricos, o que implicará redução dos gastos com medicamentos, uma das barreiras mais importantes para o acesso.

Verificou-se que de 2002 para 2012 houve importante e significativo aumento na utilização, aceitação e conhecimento dos medicamentos genéricos, enquanto a percepção quanto ao menor preço e qualidade equivalente se manteve elevada, ainda que tenha acontecido aumento na proporção de pessoas que consideram os genéricos de qualidade pior do que os medicamentos de referência. 


\section{Colaboradores}

M. C. Guttier realizou a coleta dos dados, organização e análise dos resultados e redação do artigo. M. P. T. Silveira contribuiu na análise estatística, redação do artigo, interpretação dos dados e revisão crítica do conteúdo.V. L. Luiza contribuiu na redação do artigo, interpretação dos dados e revisão crítica do conteúdo. A. D. Bertoldi participou da concepção do estudo, análise dos dados, redação do artigo, revisão crítica do conteúdo e aprovação da versão final do manuscrito.

\section{Referências}

1. Babar ZUD, Kan SW, Scahill S. Interventions promoting the acceptance and uptake of generic medicines: a narrative review of the literature. Health Policy 2014; 117:285-96.

2. Organización Mundial de la Salud. Informe sobre la salud en el mundo 2000: mejorar el desempeño de los sistemas de salud. Geneva: Organización Mundial de la Salud; 2000.

3. Sheppard A. Generic medicines: essential contributors to the long-term health of society. http:// www.imshealth.com/imshealth/Global/Content/ Document/Market_Measurement_TL/Generic Medicines_GA.pdf (acessado em 14/Set/2014).

4. Brasil. Lei Federal no 9.787, de 10 de fevereiro de 1999. Altera a Lei no 6.360 , de 23 de setembro de 1976, que dispõe sobre a vigilância sanitária e estabelece o medicamento genérico, dispõe sobre a utilização de nomes genéricos em produtos farmacêuticos e dá outras providências. Diário Oficial da União 1999; 11 fev.

5. Bertoldi AD, Barros AJD, Hallal PC. Generic drugs in Brazil: known by many, used by few. Cad Saúde Pública 2005; 21:1808-15.

6. Vosgerau MZS, Souza RKT, Soares DA. Utilização de genéricos em área de atuação da equipe de Saúde da Família em município do sul do Brasil. Rev Bras Epidemiol 2011; 14:253-63.

7. Blatt CR, Trauthman SC, Schmidt EH, Marchesan S, da Silva LM, Martins JL. Conhecimento popular e utilização dos medicamentos genéricos na população do município de Tubarão, SC. Ciênc Saúde Coletiva 2012; 17:79-87.

8. Hassali MA, Shafie AA, Jamshed S, Ibrahim MI, Awaisu A. Consumers' views on generic medicines: a review of the literature. Int J Pharm Pract 2009; 17:79-88.

\section{Agradecimentos}

À Coordenação de Aperfeiçoamento de Pessoal de Nível Superior (Capes), pela bolsa de estudos concedida à M. C. Guttier e pelo apoio financeiro para realização da pesquisa.
9. Rocha CE, Barros JAC, Silva MDP. Levantamento de dados sobre o conhecimento e informação acerca dos medicamentos genéricos em uma população de pacientes do serviço de saúde ambulatorial do Recife, Pernambuco, Brasil. Cad Saúde Pública 2007; 23:1141-50.

10. WHO Collaborating Centre for Drug Statistics Methodology. Guidelines for ATC classification and DDD assignment 2014. Oslo: WHO Collaborating Centre for Drug Statistics Methodology; 2013.

11. Associação Brasileira de Empresas de Pesquisa. Critério de Classificação Econômica Brasil. São Paulo: Associação Brasileira de Empresas de Pesquisa; 2010

12. Associação Nacional de Empresas de Pesquisa. Critério de Classificação Econômica Brasil. São Paulo: Associação Nacional de Empresas de Pesquisa; 1996.

13. Bertoldi AD, Helfer AP, Camargo AL, Tavares NUL, Kanavos P. Is the Brazilian Pharmaceutical policy ensuring population access to essential medicines? Global Health 2012; 8:6.

14. Miranda ES, Pinto CDBS, Reis ALA, Emmerick ICM, Campos MR, Luiza VL, et al. Disponibilidade no setor público e preços no setor privado: um perfil de medicamentos genéricos em diferentes regiões do Brasil. Cad Saúde Pública 2009; 25:2147-58.

15. Dias CRC, Romano-Lieber NS. Processo de implantação da política de medicamentos genéricos no Brasil. Cad Saúde Pública 2006; 22:1661-9.

16. Associação Brasileira das Indústrias de Medicamentos Genéricos. Mercado dos medicamentos genéricos, 2015. http://www.progenericos.org.br/ index.php/mercado (acessado em 03/Set/2015). 
17. Vieira FS, Zucchi P. Diferenças de preços entre medicamentos genéricos e de referência no Brasil. Rev Saúde Pública 2006; 40:444-9.

18. Agência Nacional de Vigilância Sanitária. Resolução da Diretoria Colegiada (RDC) no 17, de 2 de março de 2007. Diário Oficial da União 2007; 5 mar.

19. Agência Nacional de Vigilância Sanitária. Resolução da Diretoria Colegiada (RDC) no 134, de 29 de Maio de 2003. Diário Oficial da União 2003; 25 set.

20. Nishijima M, Biasoto Jr. G, Lagroteria E. A competição no mercado farmacêutico brasileiro após uma década de medicamentos genéricos: uma análise de rivalidade em um mercado regulado. Economia e Sociedade 2014; 23:155-86.

21. Vieira FP, Rediguieri CF, Rediguieri CF. A regulação de medicamentos no Brasil. Porto Alegre: Editora Artmed; 2013.

22. Rickwood S, Kleinrock M, Nunez-Gaviria M. The global use of medicines: outlook through 2017. Danbury: IMS Institute for Healthcare Informatics, IMS Health; 2013.

23. Chiavegatto Filho ADP, Wang Y-P, Malik AM, Takaoka J, Viana MC, Andrade LH. Determinants of the use of health care services: multilevel analysis in the Metropolitan Region of Sao Paulo. Rev Saúde Pública 2015; 49:1-12.

24. Mendoza-Sassi R, Béria JU. Utilización de los servicios de salud: una revisión sistemática sobre los factores relacionados. Cad Saúde Pública 2001; 17:819-32.

25. Costa KS, Barros MBA, Francisco PMSB, César CLG, Goldbaum M, Carandina L. Utilização de medicamentos e fatores associados: um estudo de base populacional no Município de Campinas, São Paulo, Brasil. Cad Saúde Pública 2011; 27:649-58.

26. Silva RM, Caetano R. Gastos da Secretaria Municipal de Saúde do Rio de Janeiro, Brasil, com medicamentos: uma análise do período 2002-2011. Cad Saúde Pública 2014; 30:1207-18.

27. Bertoldi AD, Helfer AP, Camargo AL, Tavares NUL, Kanavos P. Medicine prices, availability and affordability in Southern Brazil: a study of public and private facilities. London: London School of Economics and Political Science; 2010. (LSE Health Working Paper, 18/2010).

28. Sewell K, Andreae S, Luke E, Safford MM. Perceptions of and barriers to use of generic medications in a rural African American population, Alabama, 2011. Prev Chronic Dis 2012; 9:E142.

29. Helfer AP, Camargo AL, Tavares NUL, Kanavos P, Bertoldi AD. Capacidade aquisitiva e disponibilidade de medicamentos para doenças crônicas no setor público. Rev Panam Salud Pública 2012; 31:225-32.

30. Brasil. Lei no 10.858, de 13 de abril de 2004, que autoriza a Fundação Oswaldo Cruz (Fiocruz) a disponibilizar medicamentos mediante ressarcimento e dá outras providências. Diário Oficial da União 2004; 14 abr.

31. Ministério da Saúde. Portaria GM no 749, de 15de abril de 2009. Dispõe sobre a expansão do Programa Farmácia Popular do Brasil - Aqui Tem Farmácia Popular. Diário Oficial da União 2009; 16 abr.

32. Ministério da Saúde. Portaria no 184, de 3 de fevereiro de 2011. Diário Oficial da União 2011; 4 fev.
33. Inocencio M, De Vivo B. Acesso a medicamentos: análise das estratégias do estado para o desenvolvimento do Programa Farmácia Popular. Cadernos Gestão Pública e Cidadania 2011; 16:201-21.

34. Pinto CDBS, Miranda ES, Emmerick ICM, Costa NR, Osorio de Castro CGS. Medicine prices and availability in the Brazilian Popular Pharmacy Program. Rev Saúde Pública 2010; 44:611-9.

35. Da Motta GP, Domingues EP, Andrade MV, Chein F, Santiag FS, editors. Uma análise dos impactos econômicos do Programa Farmácia Popular do Brasil. Anais do XLI Encontro Nacional de Economia. Niterói: Associação Nacional dos Centros de Pós-graduação em Economia; 2014.

36. Bueno CS, Moreira AC, Oliveira KR. Cost of drugs used to treat cardiovascular disease in Brazil. Rev Panam Salud Pública 2012; 31:62-7.

37. Nardi EP, Ferraz MB, Pinheiro GRC, Kowalski SC, Sato EI. Perceptions of the population regarding generic drugs in Brazil: a nationwide survey. BMC Public Health 2015; 15:117.

38. Gossell-Williams M. Generic substitutions: a 2005 survey of the acceptance and perceptions of physicians in Jamaica. West Indian Med J 2007; 56: 458-63.

39. Chua GN, Hassali MA, Shafie AA, Awaisu A. A survey exploring knowledge and perceptions of general practitioners towards the use of generic medicines in the northern state of Malaysia. Health Policy 2010; 95:229-35.

40. Dunne S, Shannon B, Hannigan A, Dunne C, Cullen W. Physician and pharmacist perceptions of generic medicines: what they think and how they differ. Health Policy 2014; 116:214-23.

41. Agência Nacional de Vigilância Sanitária. Resolução RE no 899, de 29 de maio de 2003. Diário Oficial da União 2003; 2 jun.

42. Kobayashi E, Karigome H, Sakurada T, Satoh N, Ueda S. Patients' attitudes towards generic drug substitution in Japan. Health Policy 2011; 99:60-5.

43. Rathe J, Larsen P, Andersen M, Paulsen M, Jarbol $\mathrm{D}$, Thomsen J, et al. Associations between generic substitution and patients' attitudes, beliefs and experiences. Eur J Clin Pharmacol 2013; 69:1827-36.

44. King DR, Kanavos P. Encouraging the use of generic medicines: implications for transition economies. Croat Med J 2002; 43:462-9.

45. Instituto Brasileiro de Geografia e Estatística. Estudos e pesquisas: informações demográficas e socioeconômicas. uma análise das condições de vida da população brasileira 2010. Rio de Janeiro: Instituto Brasileiro de Geografia e Estatística; 2010.

46. Instituto Brasileiro de Geografia e Estatística. Censo demográfico 2010. Características da população e dos domicílios: resultados do universo. Rio de Janeiro: Instituto Brasileiro de Geografia e Estatística; 2010.

47. Drozdowska A, Hermanowski T. Predictors of generic substitution: the role of psychological, sociodemographic, and contextual factors. Res Social Adm Pharm 2015; 12:119-29.

48. Bertoldi AD, Barros AJ, Wagner A, Ross-Degnan D, Hallal PC. A descriptive review of the methodologies used in household surveys on medicine utilization. BMC Health Serv Res 2008; 8:222. 


\section{Abstract}

This study compared the perception, knowledge, and use of generic drugs by adults in Pelotas, Rio Grande do Sul State, Brazil, using two cross-sectional populationbased studies from 2002 and 2012. Study outcomes were: (a) prevalence of use of generics; (b) generics as a proportion of all medication; (c) users' perceptions of prices and quality; (d) users' knowledge of generics; and (e) strategies for acquisition of medicines. Prevalence of generics use increased from 3.6\% (95\% CI: 3.04.3) to $26.1 \%$ (95\% CI: 24.5-27.7) in the 10-year period. Perceptions of prices and quality of generics remained stable, identification of characteristics that distinguish generics from other drugs improved $(p<0.001)$, and drug classification errors decreased $(p<0.001)$. There was a significant increase in acquiring medication by replacing prescribed drugs with generics. Between 2002 and 2012 there was an increase in knowledge and use of generics, while perception of lower prices and equivalent quality remained high.

Patient Medication Knowledge; Drug Substitution; Generic Drugs

\section{Resumen}

Este estudio compara la percepción, conocimiento y uso de medicamentos genéricos en adultos de Pelotas, Río Grande do Sul, Brasil, a través de dos estudios transversales de base poblacional, realizados en 2002 y 2012. Los resultados estudiados fueron: (a) prevalencia de utilización de medicamentos genéricos; (b) proporción de uso de medicamentos genéricos entre los demás medicamentos; (c) percepción de los usuarios sobre el precio y calidad de los medicamentos genéricos; (d) conocimiento de los usuarios sobre medicamentos genéricos y (e) estrategias de adquisición de medicamentos. La prevalencia de uso de medicamentos genéricos aumentó de 3,6\% (IC95\%: 3,0-4,3) a 26,1\% (IC95\%: 24,5-27,7) en un período de 10 años. La percepción sobre el precio y calidad de los medicamentos genéricos se mantuvo estable, la identificación de las características que diferencian los medicamentos genéricos de los demás medicamentos mejoró ( $p<$ $0,001)$ y el error de clasificación de medicamentos disminuyó ( $p<0,001)$. Hubo un aumento significativo en la estrategia de adquisición de medicamentos, a través de la sustitución del medicamento prescrito por el medicamento genérico. Entre 2002 y 2012 aumentó el conocimiento y uso de medicamentos genéricos, mientras que la percepción en lo referente al menor precio y calidad equivalente, se mantuvieron elevadas.

Conocimiento de la Medicación por el Paciente; Sustitución de Medicamentos; Medicamentos Genéricos
Recebido em 04/Mai/2015

Versão final reapresentada em 26/Out/2015

Aprovado em 22/Fev/2016 